\title{
THE RATE OF INCREASE OF REAL CONTINUOUS SOLUTIONS OF CERTAIN ALGEBRAIC FUNCTIONAL EQUATIONS
}

\author{
BY \\ K. L. COOKE
}

1. Introduction. The problem discussed in this paper is that of describing the rate of increase, as $t$ becomes indefinitely large, of real solutions of algebraic functional equations of the form

$$
P(t, u(t), u(t+\delta(t)))=0 .
$$

In this equation, and throughout this paper, $P(t, u, v)$ denotes a polynomial in the variables $t, u, v$, with real coefficients, and $\delta(t)$ is a function which is positive and continuous for $t$ greater than or equal to some positive number $a$. The results presented below generalize those of O. E. Lancaster [4], and S. M. Shah $[6 ; 7]$, who discussed the case in which $\delta(t)$ is constant for $t \geqq a$. Their work, in turn, was a natural sequel to the striking results of Borel [1], and Lindelöf [5], on algebraic differential equations $\left({ }^{1}\right)$.

For convenience in stating the results obtained in this paper, let us introduce the following definition.

A real function $u(t)$ will be said to be a continuous solution of an equation (1.1) if there exists a number $b \geqq a$ such that $u(t)$ is continuous and is a solution of (1.1) for all $t \geqq b$.

The main object of this paper is to show that, provided $\delta(t)$ is positive and continuous for $t \geqq a$ and provided $t+\delta(t)$ is monotonic increasing for $t \geqq a$, every continuous solution of an equation of the form in (1.1) must satisfy a relation of the form

$$
\liminf _{t \rightarrow+\infty} \frac{|u(t)|}{f(t)}=0,
$$

and every continuous monotonic $\left({ }^{2}\right)$ solution must satisfy

$$
\lim _{t \rightarrow+\infty} \frac{|u(t)|}{f(t)}=0 .
$$

Here $f(t)$ is a certain positive, continuous, nondecreasing function which de-

Presented to the Society November 1, 1957; received by the editors November 30, 1957.

(1) Another extension of this work was recently given by the author in an article [2], on algebraic differential-difference equations.

(2) Montonicity can be replaced by a weaker condition, but this has not been done here because the condition seems less natural than monotonicity. For an indication of the nature of the weaker condition, refer to Shah [6], Theorem 2. 
pends on $P$ and $\delta$, but not on the particular solution $u$ in question. The actual form of $f(t)$ is discussed in Theorems 2 and 4 below. Roughly speaking, then, there is a maximal possible rate of growth of a solution of (1.1), this maximal rate being determined solely by the form of the equation.

The relations in (1.2) and (1.3) contrast sharply with Lindelöf's result on solutions of algebraic differential equations of the form

$$
P\left(t, u(t), u^{\prime}(t)\right)=0 .
$$

He showed that every continuous solution $u(t)$ extending to $t=+\infty$ satisfies $\left({ }^{3}\right)$

$$
\lim _{t \rightarrow+\infty} \frac{|u(t)|}{e\left(t^{B}\right)}=0
$$

for a certain positive number $B$ depending only on $P$. This conclusion, similar to (1.3), is correct even for nonmonotonic solutions of (1.4). We shall show below that for solutions of (1.1), on the other hand, the "lim inf" in (1.2) cannot be replaced by "lim," unless a restrictive hypothesis, such as monotonicity, is imposed on the solution $u$.

The "best possible" form of $f(t)$ is found in this paper for three special classes of equations. The first, the class of first order algebraic difference equations

$$
P(t, u(t), u(t+1))=0,
$$

is obtained by putting $\delta(t)=1$ in (1.1), and was the class of equations considered by Shah. We shall show in $\S 4$ that for this class of equations, (1.2) and (1.3) hold with $f(t)=e_{2}(B t)$, where $B$ is a positive number depending only on $P$. Shah proved that (1.2) is a best possible result in two senses. First, it is not possible to replace "lim inf" by "lim" without restrictive hypothesis on $u(t)$. Second, the denominator $e_{2}(B t)$ cannot be replaced by a function of slower rate of growth; for example, $e_{2}(t \log n)=e\left(n^{t}\right)$ is a solution of

$$
u(t+1)-u(t)^{n}=0,
$$

which is an equation of the form in (1.5) if $n$ is a positive integer. This example, by the way, indicates that no improvement in the results is possible even for equations in which $t$ does not explicitly appear.

The second special class of equations is the class of first order algebraic $q$-difference equations $\left({ }^{4}\right)$

$$
P(t, u(t), u(q t))=0,
$$

obtained from (1.1) by putting $\delta(t)=(q-1) t$. We shall show in $\S 4$ that for

(3) We shall employ the notation $e_{0}(t)=t, e(t)=e_{1}(t)=e^{t}, e_{k}(t)=\exp \left(e_{k-1}(t)\right)$, which was adopted by G. H. Hardy [3].

(4) We shall show in $\$ 4$ that the same conclusions are valid if $0<g<1$. 
this class of equations, (1.2) and (1.3) hold with $f(t)=e\left(t^{B}\right)$, where $B$ is a positive number depending only on $P$ and $q$, and that these results are also best possible.

The last special class of equations considered in this paper is obtained from (1.1) by putting $\delta(t)=t^{q}-t(q>1)$. The equations have the form

$$
P\left(t, u(t), u\left(t^{a}\right)\right)=0, \quad(q>1),
$$

and will be called qth power functional equations. We shall show in $\$ 4$ that for this class of equations, (1.2) and (1.3) hold with $f(t)=e\left((\log t)^{B}\right)$, where $B$ is a positive number depending only on $P$ and $q$, and that these results are best possible.

In the concluding section of this article, a few brief remarks are made concerning possible extensions and generalizations.

2. Lemmas. Let us suppose that $\delta(t)$ is positive and continuous and that $t+\delta(t)$ is monotonic increasing for $t \geqq a$. Let $b$ be any fixed number at least as large as $a$. Define

$$
\begin{aligned}
b_{0} & =b, \\
b_{n+1} & =b_{n}+\delta\left(b_{n}\right), \quad(n=0,1,2 \cdots) .
\end{aligned}
$$

The number $b_{n}$ will be called the $n t h \delta$-image of $b$. Since $\delta(t)$ is positive for $t \geqq a$, the $\delta$-images of $b$ form an increasing sequence. If this sequence were to approach a finite limit $L$, we should have $L>a$ and

$$
L=\lim _{n \rightarrow \infty} b_{n+1}=\lim _{n \rightarrow \infty}\left\{b_{n}+\delta\left(b_{n}\right)\right\}=L+\delta(L) ;
$$

this is impossible since $\delta(L)>0$. Hence the sequence of $\delta$-images of any number $b$ increases monotonically to $+\infty$.

Let the symbol $I_{n}(b)$ denote the interval $\left[b_{n}, b_{n+1}\right)(n=0,1,2, \cdots)$. $I_{n}(b)$ will be called the $n$th $\delta$-image of the interval $I_{0}(b) \equiv[b, b+\delta(b))$. Since $t+\delta(t)$ has been supposed to be monotonic increasing, the mapping

$$
M: t \rightarrow t+\delta(t)
$$

is a continuous, one-to-one mapping of each $I_{j}(b)$ onto $I_{j+1}(b)$, and $M^{n}$ is a continuous, one-to-one mapping of $I_{j}(b)$ onto $I_{n+j}(b)(j=0,1,2, \cdots$; $n=1,2, \cdots)$.

We can now state the first of four lemmas we shall require.

Lemma 1. Let $\delta(t)$ be positive and continuous and let $t+\delta(t)$ be monotonic increasing for $t \geqq a$. Let $A$ be a positive number and let $f(t)$ be a function which is positive and continuous for $t \geqq a$ and which satisfies $\left(^{5}\right)$ the following growth property:

$\left(\mathrm{G}_{1}\right)$ Given any $b \geqq a$, any $w>1$, and an arbitrary sequence of points $\left\{\tau_{n}\right\}$ having $\tau_{n}$ in the nth $\delta$-image interval $I_{n}(b)$,

(5) The existence of such functions $f(t)$ is demonstrated in $\S 3$ below. 


$$
\lim _{n \rightarrow \infty} \frac{w^{A^{n}}}{f\left(\tau_{n}\right)}=0
$$

Then it follows that to each function $u(t)$ which is continuous and non-negative for $t \geqq a$ and which satisfies

$$
\limsup _{t \rightarrow+\infty} \frac{u(t)}{f(t)}>0
$$

there corresponds a sequence $\left\{s_{n}\right\}$ such that $s_{n} \geqq a(n=1,2, \cdots), s_{n} \rightarrow+\infty$ as $n \rightarrow \infty$, and

$$
u(t+\delta(t)) \geqq u(t)^{A} \quad \text { for } t=s_{n} \quad(n=1,2, \cdots) .
$$

Proof. Let $u(t)$ be a continuous, non-negative function which satisfies (2.2). There is a positive number $D$ and there is an increasing sequence $\left\{\xi^{i}\right\}(i=1,2, \cdots)$ such that $\xi^{i} \rightarrow+\infty$ as $i \rightarrow \infty$ and

$$
\lim _{i \rightarrow \infty} \frac{u\left(\xi^{i}\right)}{f\left(\xi^{i}\right)}=D
$$

Suppose the lemma is false. Then there is a number $b \geqq a$ such that

$$
u(t+\delta(t))<u(t)^{A} \quad \text { for all } t \geqq b .
$$

Let $x^{i}$ denote the unique point in $I_{0}(b)$ whose image under some number of repetitions of the mapping $M$ is $\xi^{i}$. Let $n_{i}$ be the appropriate number of repetitions. Let $\left\{x_{j}^{i}\right\}\left(j=0,1,2, \cdots ; x_{0}^{i}=x^{i}\right)$ be the sequence of $\delta$-images of $x^{i}$. Then from (2.5) we have, by induction,

$$
u\left(x_{n_{i}}^{i}\right)<u\left(x_{0}^{i}\right)^{A^{n_{i}}} .
$$

Let $S$ be the supremum of $u(t)$ for $t$ in $I_{0}(b)$, and take $w>\max (S, 1)$. Then, since $x_{n_{i}}^{i}=\xi^{i}$ and $x_{0}^{i}=x^{i}$, we obtain

$$
\frac{u\left(\xi^{i}\right)}{f\left(\xi^{i}\right)}<\frac{u\left(x^{i}\right)^{A^{n_{i}}}}{f\left(x_{n_{i}}^{i}\right)}<\frac{w^{A^{n_{i}}}}{f\left(x_{n_{i}}^{i}\right)}, \quad i=1,2, \cdots
$$

Since $n_{i} \rightarrow \infty$ as $i \rightarrow \infty$, and $x_{n_{i}}^{i}$ lies in the $n_{i}$ th $\delta$-image interval of $I_{0}(b)$, it follows from property $\left(\mathrm{G}_{1}\right)$ that

$$
\lim _{i \rightarrow \infty} \frac{u\left(\xi^{i}\right)}{f\left(\xi^{i}\right)}=0
$$

which contradicts (2.4). This proves Lemma 1.

The second important lemma follows.

LEMma 2. Let $\delta(t)$ be positive and continuous and let $t+\delta(t)$ be monotonic increasing for $t \geqq a$. Let $A$ be a positive number and let $f(t)$ be a function which 
is positive, continuous, and nondecreasing for $t \geqq a$ and which satisfies the growth property $\left(\mathrm{G}_{1}\right)$ stated in Lemma 1. Let $g(t)$ be a function which is continuous for $t \geqq a$ and which satisfies

$$
\begin{gathered}
g(t)>1 \quad \text { if } t \geqq a, \\
\lim _{t \rightarrow+\infty} \frac{g(t)}{f(t)}=0,
\end{gathered}
$$

and

$\left(\mathrm{G}_{2}\right)$ Given any positive number $\beta$ there exists a number $T=T(\beta), T \geqq a$, such that

$$
\frac{g(d)^{A^{m+1}}}{f\left(d_{m}\right)} \leqq \beta
$$

for every number $d \geqq T$ and every integer $m \geqq 0$. Then it follows that to each function $u(t)$ which is continuous, non-negative, and nondecreasing for $t \geqq a$ and which satisfies

$$
\limsup _{t \rightarrow+\infty} \frac{u(t)}{f(t)}>0
$$

there corresponds a sequence $\left\{s_{n}\right\}$ such that $s_{n} \geqq a(n=1,2, \cdots), s_{n} \rightarrow+\infty$ as $n \rightarrow \infty$,

$$
\begin{array}{rlrl}
u(t+\delta(t)) & \geqq u(t)^{A} & \text { for } t & =s_{n}(n=1,2, \cdots), \\
u(t) & \geqq g(t) & \text { for } t=s_{n}(n=1,2, \cdots) .
\end{array}
$$

Proof. Let

$$
D=\limsup _{t \rightarrow+\infty} \frac{u(t)}{f(t)} .
$$

Choose any $\beta$ and choose any $C$ for which $0<\beta<C<D$. If $u(t) \geqq g(t)$ for all large $t$, the result follows from Lemma 1. Suppose, therefore, that $u(t)<g(t)$ for a sequence of values of $t$ tending to infinity. Let $b$ be an arbitrarily large number in this sequence, so that $u(b)<g(b)$, and suppose $b \geqq T(\beta)$. From (2.10), there is a number $c, c>b$, such that $u(c)>C f(c)$. Furthermore, by (2.7) we may suppose that $b$ is so large that $g(t)<C f(t)$ for $t \geqq b$. Then

$$
u(c)>C f(c)>g(c) \text {. }
$$

Since $u(t) / g(t)$ is continuous, there is a largest number $d$ such that $b<d<c$ and $u(d) / g(d)=1$. Note that $u(t) \geqq g(t)$ for $d \leqq t \leqq c$. Thus there are intervals $[d, c]$ with $d$ arbitrarily large in which (2.9) holds. To complete the proof of the lemma, we need only show that in each such interval there is a point $t$ at which (2.3) also holds. Consider the sequence of images $d_{n}$ of $d_{0}=d$, 
and let $\left\{I_{n}(d)\right\}$ be the corresponding sequence of image intervals. The number $c$ will fall within exactly one such interval, say $I_{m}(d)(m \geqq 0)$, so that $d_{m} \leqq c<d_{m+1}$. Since $u(t)$ is nondecreasing, (2.11) yields $u\left(d_{m+1}\right) \geqq u(c)>C f(c)$. Since $f(t)$ is also nondecreasing,

$$
u\left(d_{m+1}\right)>C f\left(d_{m}\right) .
$$

Now suppose that

$$
u\left(d_{j}\right)<u\left(d_{j-1}\right)^{A} \quad(j=1,2, \cdots, m+1) .
$$

It readily follows that

$$
u\left(d_{m+1}\right)<u(d)^{A^{m+1}} .
$$

Combining this inequality with that in (2.12), and replacing $u(d)$ by $g(d)$, we find

$$
\frac{g(d)^{A^{m+1}}}{f\left(d_{m}\right)}>C .
$$

Inasmuch as $C>\beta$ and $d \geqq T(\beta)$, this contradicts the growth property $\left(\mathrm{G}_{2}\right)$. Consequently there must be at least one integer $j, 1 \leqq j \leqq m+1$, for which (2.13) is false, that is for which

$$
u\left(d_{j}\right) \geqq u\left(d_{j-1}\right)^{A} .
$$

Thus (2.3) holds for $t=d_{j-1}$; also (2.9) holds for $t=d_{j-1}$, since $d_{j-1}$ lies in $[d, c]$. This completes the proof of Lemma 2 .

For the sake of later convenience, we also call attention to the following simple fact.

Lemma 3. If

$$
\lim _{t \rightarrow+\infty} \psi(t)=+\infty
$$

and

$$
\lim _{t \rightarrow+\infty} \frac{\phi(t)}{\psi(t)}=0,
$$

and if $k$ is any positive integer, then

$$
\lim _{t \rightarrow+\infty} \frac{e_{k}(\phi(t))}{e_{k}(\psi(t))}=0 .
$$

This lemma is readily proved by induction on $k$, since

$$
\frac{e_{k}(\phi(t))}{e_{k}(\psi(t))}=e\left\{-e_{k-1}(\psi(t))\left[1-\frac{e_{k-1}(\phi(t))}{e_{k-1}(\psi(t))}\right]\right\}, \quad k=1,2,3, \cdots
$$


Lemma 4. Suppose that $1<A<\alpha$ and that $\phi(t)$ is positive for all sufficiently large $t$. Suppose also that

$$
\lim _{t \rightarrow+\infty} \psi(t)=+\infty
$$

and

$$
\lim _{t \rightarrow+\infty} \frac{\phi(t)}{\psi(t)}=0 .
$$

Then, given any positive number $\beta$, there exists a positive number $T=T(\beta)$ such that

$$
\frac{e\left\{A^{m+1} \phi(t)\right\}}{e\left\{\alpha^{m} \psi(t)\right\}} \leqq \beta
$$

for every $t \geqq T$ and every integer $m \geqq 0$.

Proof. From Lemma 3 it is clear that

$$
\lim _{t \rightarrow+\infty} \frac{e\{A \phi(t)\}}{e\{\psi(t)\}}=0 .
$$

Therefore there exists a $T=T(\beta)$ such that $\phi(t)>0$ for $t \geqq T$ and such that

$$
A \phi(t)-\psi(t) \leqq \min (0, \log \beta) \quad \text { for } t \geqq T \text {. }
$$

Let

$$
h_{m}(t)=A^{m+1} \phi(t)-\alpha^{m} \psi(t) .
$$

Since $1<A<\alpha$ and $\phi(t)>0$,

$$
h_{m+1}(t)-h_{m}(t)<\alpha^{m}(\alpha-1)[A \phi(t)-\psi(t)] .
$$

From (2.15) we can now deduce that for each $t \geqq T,\left\{h_{m}(t)\right\}$ is a nonincreasing sequence and therefore also

$$
h_{m}(t) \leqq h_{0}(t) \leqq \log \beta \quad(m \geqq 0) .
$$

Therefore $e\left\{h_{m}(t)\right\} \leqq \beta$ for $t \geqq T, m \geqq 0$. This is what was to be proved.

3. General theorems. In this section we shall state and prove the general theorems, on the magnitude of solutions of the equation (1.1), to which we alluded in the Introduction.

THEOREM 1. Consider any equation of the form

$$
P(t, u(t), u(t+\delta(t)))=0,
$$

where $\delta(t)$ is positive and continuous and $t+\delta(t)$ is increasing for $t \geqq a$. There exist positive numbers $A$ and $C$, depending only on the polynomial $P$ (not on $u$ or $\delta)$, such that if $f(t)$ satisfies the two hypotheses listed below, then 


$$
\liminf _{t \rightarrow+\infty} \frac{|u(t)|}{f(t)}=0
$$

for every continuous solution $u(t)$ of (3.1). The two hypotheses are:

$\left(\mathrm{H}_{1}\right) f(t)$ is a positive, continuous function for $t \geqq a$ satisfying

$$
\lim _{t \rightarrow+\infty} \frac{t^{c}}{f(t)}=0 .
$$

$\left(\mathrm{H}_{2}\right)$ Given any $b \geqq a$, any $w>1$, and an arbitrary sequence of points $\left\{\tau_{n}\right\}$ having $\tau_{n}$ in the nth $\delta$-image interval $I_{n}(b)$,

$$
\lim _{n \rightarrow \infty} \frac{w^{A^{n}}}{f\left(\tau_{n}\right)}=0 .
$$

Proof. The equation in (3.1) can be written in the form

$$
\sum_{i=0}^{I} \sum_{j=0}^{J} \sum_{k=0}^{K} T_{i j k}=0
$$

where

$$
T_{i j k}=a_{i j k} t^{i} u(t)^{j} u(t+\delta(t))^{k} .
$$

The $a_{i j k}$ are real numbers independent of $t$. Among the terms $T_{i j k}$ there is exactly one term $T_{p q r}$ selected in the following way:

(1) Choose $r=K$.

(2) Choose $q$ to be the greatest of the values of $j$ among all the terms $T_{i j r}$.

(3) Choose $p$ to be the greatest of the values of $i$ among all the terms $T_{i q r}$.

The term $T_{p q r}$ so defined will be called the principal term.

Except for constant factors, the ratios $T_{i j k} / T_{p q r}$ are of the following three possible types (excepting the ratio $T_{p q r} / T_{p q r}$ ):

$$
\frac{t^{i-p} u(t)^{i-q}}{u(t+\delta(t))^{r-k}}
$$

where $r>k$.

$$
\frac{t^{i-p}}{u(t)^{q-j}}
$$

where $q>j$.

(c)

$$
t^{i-p}
$$

where $p>i$. Let $R$ be the least non-negative number which equals or exceeds the maximum value of $(j-q) /(r-k)$ for all ratios of type (a). Let $A$ be any 
positive number such that $A \geqq R+1$. Let $C$ be a positive number which equals or exceeds the maximum value of $(i-p) /(q-j)$ for all ratios of type (b) and which equals or exceeds the maximum value of $(i-p) /(r-k)$ for all ratios of type (a).

Now suppose $f(t)$ to be a function satisfying $\left(\mathrm{H}_{1}\right)$ and $\left(\mathrm{H}_{2}\right)$. Let $u(t)$ be any continuous solution of (3.1). We wish to show that (3.2) holds. Suppose the contrary. Then

$$
\liminf _{t \rightarrow+\infty} \frac{|u(t)|}{f(t)}>0 .
$$

This, together with (3.3), implies that

$$
\lim _{t \rightarrow+\infty} \frac{t^{C}}{|u(t)|}=0 .
$$

Since $\left(\mathrm{H}_{2}\right)$ is exactly the growth condition $\left(\mathrm{G}_{1}\right)$ of Lemma 1, and (3.6) implies (2.2), it follows from Lemma 1 that there is a sequence $\left\{s_{n}\right\}$ for which $s_{n} \rightarrow+\infty$ as $n \rightarrow \infty$ and for which

$$
|u(t+\delta(t))| \geqq|u(t)|^{A} \quad \text { for } t=s_{n}(n=1,2, \cdots) .
$$

For each value $t=s_{n}$, the function $u(t)$ satisfies not only the equation in (3.1), but also the equation

$$
\sum_{i=0}^{I} \sum_{j=0}^{J} \sum_{k=0}^{K} \frac{T_{i j k}}{T_{p q r}}=0
$$

For every $t \geqq a$,

$$
\left|\frac{t^{i-p} u(t)^{j-q}}{u(t+\delta(t))^{r-k}}\right|=\left\{\frac{t^{i-p / r-k}|u(t)|^{j-q / r-k}}{|u(t+\delta(t))|}\right\}^{r-k} .
$$

For ratios of type (a), $r>k$. We now set $t=s_{n}$. Since $u(t)$ becomes infinite as $n \rightarrow \infty$, by (3.7), the above expression cannot exceed

$$
\left\{\frac{t^{C}|u(t)|^{R}}{|u(t+\delta(t))|}\right\}^{r-k}, \quad t=s_{n}, r>k,
$$

if $n$ is sufficiently large. By (3.8) and the fact that $A \geqq R+1$, this is no greater than

$$
\left\{\frac{t^{C}}{|u(t)|}\right\}^{r-k}
$$

From (3.7) it now follows that all ratios of type (a) approach zero, at the points $t=s_{n}$, as $n \rightarrow \infty$. For ratios of type (b) we have 


$$
\left|\frac{t^{i-p}}{u(t)^{q-i}}\right|=\left\{\frac{t^{i-p / q-j}}{|u(t)|}\right\}^{q-i} \leqq\left\{\frac{t^{C}}{|u(t)|}\right\}^{q-i}, \quad\left(t=s_{n}\right) .
$$

Since $q>j$, (3.7) again shows that all ratios of type (b) approach zero as $t=s_{n} \rightarrow+\infty$. It is clear that all ratios of type (c) approach zero.

It now follows that we can find a positive integer $N$ such that the sum of all ratios $T_{i j k} / T_{p q r}$ is less than one in absolute value when $t=s_{N}$, whereas $T_{p q r} / T_{p q r}=1$. Thus (3.9) cannot be satisfied at the point $s_{N}$. This contradiction shows that (3.2) is satisfied, as was to be proved.

TheOREM 2. Consider any equation of the form

$$
P(t, u(t), u(t+\delta(t)))=0,
$$

where $\delta(t)$ is positive and continuous and $t+\delta(t)$ is increasing for $t \geqq a$. Then there exists a positive, continuous function $f(t)$, depending on $P$ and $\delta$, such that every continuous solution $u(t)$ of (3.1) satisfies

$$
\liminf _{t \rightarrow+\infty} \frac{|u(t)|}{f(t)}=0
$$

Moreover, if the sequence $\left\{a_{n}\right\}$ of $\delta$-images of $a$ is of such a nature that

$$
\lim _{n \rightarrow \infty} \frac{n}{e_{\nu}\left(a_{n}\right)}=0
$$

for some non-negative integer $\nu$, then $f(t)$ may be taken to be $e_{\nu+2}(t)$.

Proof. Let $A$ and $C$ be the numbers whose existence was asserted in Theorem 1 . Let $F(t)$ be any function which satisfies the following:

(i) $F(t)$ is positive, continuous, and nondecreasing for $t \geqq a$;

$$
\begin{aligned}
\lim _{n \rightarrow \infty} \frac{n}{F\left(a_{n}\right)} & =0, \\
\lim _{t \rightarrow \infty} \frac{t^{C}}{e_{2}\{F(t)\}} & =0 .
\end{aligned}
$$

It is clear that such a function exists. Define $f(t)=e_{2}\{F(t)\}$. By (i) and (iii), $f(t)$ satisfies $\left(\mathrm{H}_{1}\right)$ of Theorem 1 . Moreover, let there be given any $b \geqq a$, any $w>1$, and any sequence $\left\{\tau_{n}\right\}$ with $\tau_{n}$ in $I_{n}(b)$. Since $\tau_{n} \geqq b_{n} \geqq a_{n}$ and since $F(t)$ is nondecreasing,

$$
\frac{w^{A^{n}}}{f\left(\tau_{n}\right)}=\frac{e_{2}\{n \log A+\log \log w\}}{e_{2}\left\{F\left(\tau_{n}\right)\right\}} \leqq \frac{e_{2}\{n \log A+\log \log w\}}{e_{2}\left\{F\left(a_{n}\right)\right\}} .
$$

By (ii), 


$$
\lim _{n \rightarrow \infty} \frac{n \log A+\log \log w}{F\left(a_{n}\right)}=0 .
$$

It follows from Lemma 3 that $f(t)$ satisfies the hypothesis $\left(\mathrm{H}_{2}\right)$ in Theorem 1 . Therefore every continuous solution $u(t)$ must satisfy (3.2).

If for some non-negative integer $\nu,(3.10)$ holds, then $F(t)=e_{\nu}(t)$ satisfies (i), (ii), and (iii), and (3.2) is valid with $f(t)=e_{\nu+2}(t)$.

The next two theorems deal with monotonic solutions of (3.1). It will be noted that for such solutions a result much more precise than that in (3.2) can be given.

Theorem 3. Consider any equation of the form

$$
P(t, u(t), u(t+\delta(t)))=0,
$$

where $\delta(t)$ is positive and continuous and $t+\delta(t)$ is increasing for $t \geqq a$. There exist positive numbers $A$ and $C$, depending only on the polynomial $P$ (not on $u$ or $\delta)$, such that if $f(t)$ satisfies the three hypotheses listed below, then

$$
\lim _{t \rightarrow+\infty} \frac{|u(t)|}{f(t)}=0
$$

for every continuous monotonic solution of (3.1). The hypotheses are:

$\left(\mathrm{H}_{3}\right)$ The function $f(t)$ is positive, continuous, and nondecreasing for $t \geqq a$. Also, given any $b \geqq a$, any $w>1$, and an arbitrary sequence of points $\left\{\tau_{n}\right\}$ having $\tau_{n}$ in the $n$th $\delta$-image interval $I_{n}(b)$,

$$
\lim _{n \rightarrow \infty} \frac{w^{A^{n}}}{f\left(\tau_{n}\right)}=0 .
$$

$\left(\mathrm{H}_{4}\right)$ There is a positive, continuous function $\mathrm{g}(t)$ for $t \geqq a$ satisfying

$$
\lim _{t \rightarrow+\infty} \frac{g(t)}{f(t)}=0
$$

and

$$
\lim _{t \rightarrow+\infty} \frac{t^{C}}{g(t)}=0
$$

$\left(\mathrm{H}_{5}\right)$ Given any positive number $\beta$, however small, there exists a number $T=T(\beta), T \geqq a$, such that

$$
\frac{g(t)^{A^{m+1}}}{f\left(t_{m}\right)} \leqq \beta
$$

for every number $t \geqq T$ and every integer $m \geqq 0$.

Proof. Choose $r, q$, and $p$ and also $R, A$, and $C$ as in the proof of Theorem 
1. Suppose $f(t)$ and $g(t)$ to be functions satisfying the three hypotheses stated above. Let $u(t)$ be any continuous monotonic sulution of (3.1). We wish to show that (3.11) holds. Suppose the contrary. Then

$$
\limsup _{t \rightarrow+\infty} \frac{|u(t)|}{f(t)}>0 .
$$

(3.12) and (3.13) show that $f(t) \rightarrow+\infty$ as $t \rightarrow+\infty$. From (3.15) it follows that the monotonic function $u(t)$ is either increasing to $+\infty$ or decreasing to $-\infty$. Furthermore, Lemma 2 may be applied. Therefore there is a sequence $\left\{s_{n}\right\}$ for which $s_{n} \rightarrow+\infty$ as $n \rightarrow \infty$ and for which

$$
\begin{aligned}
|u(t+\delta(t))| & \geqq|u(t)|^{A} & & \text { for } t=s_{n}(n=1,2, \cdots), \\
|u(t)| & \geqq g(t) & & \text { for } t=s_{n}(n=1,2, \cdots) .
\end{aligned}
$$

As in the proof of Theorem 1, we find that ratios of types (a) and (b) are bounded by

$$
\left\{\frac{t^{C}}{|u(t)|}\right\}^{r-k} \text { and }\left\{\frac{t^{c}}{|u(t)|}\right\}^{q-j},
$$

respectively, at the points $t=s_{n}$. Since $|u(t)| \geqq g(t)$, these bounds may be replaced by

$$
\left\{\frac{t^{c}}{g(t)}\right\}^{r-k} \text { and }\left\{\frac{t^{c}}{g(t)}\right\}^{q-i} \text {. }
$$

Since $r>k$ for all ratios of type (a) and $q>j$ for all ratios of type (b), it follows from (3.13) that all such ratios approach zero as $t=s_{n} \rightarrow+\infty$. It is again clear that all ratios of type (c) approach zero. We therefore reach the same contradiction that we reached in Theorem 1. Consequently (3.11) must hold.

TheOREM 4. Consider any equation of the form

$$
P(t, u(t), u(t+\delta(t)))=0,
$$

where $\delta(t)$ is positive and continuous and $t+\delta(t)$ is increasing for $t \geqq a$. Then there exists a positive, continuous, nondecreasing function $f(t)$, depending on $P$ and $\delta$, such that every continuous monotonic solution $u(t)$ of (3.1) satisfies

$$
\lim _{t \rightarrow+\infty} \frac{|u(t)|}{f(t)}=0 .
$$

Moreover, if the sequence $\left\{a_{n}\right\}$ of $\delta$-images of $a$ is of such a nature that

$$
\lim _{n \rightarrow \infty} \frac{n}{e_{\nu}\left(a_{n}\right)}=0
$$

and 


$$
\frac{n}{e_{\nu}\left(a_{n}\right)} \leqq \frac{1}{e_{\nu}(a)} \quad \text { for } n=0,1,2, \cdots,
$$

for some sufficiently large integer $\nu$, then $f(t)$ may be taken to be $e_{\nu+2}(t)$.

Proof. Let $A$ and $C$ be the numbers whose existence was asserted in Theorem 3. Let $g(t)=t^{B}$ where $B$ is any number greater than $C$. Let $F(t)$ be any function which satisfies the following:

(i) $F(t)$ is positive, continuous, and nondecreasing for $t \geqq a$;

$$
\begin{aligned}
\lim _{t \rightarrow+\infty} \frac{B \log t}{e\{F(t) / 2\}} & =0 ; \\
\lim _{n \rightarrow \infty} \frac{n}{F\left(a_{n}\right)} & =0 ; \\
F\left(a_{n}\right) & \geqq n F(a) \quad \text { for } n=0,1,2, \cdots ; \\
F(a) & >2 \log A .
\end{aligned}
$$

It is clear that such a function exists. Define $f(t)=e_{2}\{F(t)\}$. We shall show that $f(t)$ satisfies the hypotheses of Theorem 3 . With the aid of (ii) and Lemma 3 , it can be shown that $\left(\mathrm{H}_{4}\right)$ is satisfied. From (iii) it can be shown that $\left(\mathrm{H}_{3}\right)$ is satisfied, just as in the proof of Theorem 2.

It remains to prove that $\left(\mathrm{H}_{5}\right)$ is satisfied. Let $\beta>0$ be given. Since $a \leqq t \leqq t_{m}$ and $a_{m} \leqq t_{m}$, and $F$ is nondecreasing,

$$
F\left(t_{m}\right)=\left(F\left(t_{m}\right)+F\left(t_{m}\right)\right) / 2 \geqq\left(F(t)+F\left(a_{m}\right)\right) / 2
$$

and by (iv)

$$
F\left(t_{m}\right) \geqq(F(t)+m F(a)) / 2 .
$$

Hence

$$
\frac{g(t)^{A^{m+1}}}{f\left(t_{m}\right)} \leqq \frac{e\left(A^{m+1} B \log t\right)}{e_{2}\{(F(a) m+F(t)) / 2\}}
$$

If we put

$$
\begin{aligned}
\alpha & =e(F(a) / 2), \\
\phi(t) & =B \log t, \\
\psi(t) & =e(F(t) / 2),
\end{aligned}
$$

this is of the form treated in Lemma 4 . By (v), $\alpha>A$, and by the definition of $A, A>1$. Also

$$
\lim _{t \rightarrow \infty} \frac{\phi(t)}{\psi(t)}=0
$$


by (ii). Therefore Lemma 4 shows that there exists a positive number $T=T(\beta)$ such that the equation in (3.14) is satisfied for $t \geqq T$ and $m \geqq 0$. Thus $\left(\mathrm{H}_{5}\right)$ is satisfied. It follows from Theorem 3 that every continuous monotonic solution $u(t)$ of (3.1) satisfies (3.11).

If (3.10) and (3.17) hold for a non-negative integer $\nu$ sufficiently large to ensure that (v) is true, the function $F(t)=e_{v}(t)$ will satisfy (i) through (v) for this $\nu$, and we may take $f(t)=e_{\nu+2}(t)$. Of course, (v) is no real restriction on $\nu$ since we can take $a$ as large as we please.

4. Special classes of equations. In this section, we shall apply the general methods and theorems above to the study of the three special classes of equations mentioned in $\S 1$. First let us discuss algebraic difference equations of the first order. Such equations may be assumed to be of the form

$$
P(t, u(t), u(t+1))=0 .
$$

Although Theorems 2 and 4 apply to such equations, it is possible to be much more precise about the nature of $f(t)$ when considering (4.1) than when considering the general class of equations (3.1). In fact, the "best possible" form of $f(t)$ may be stated, as is done in the following theorem.

THEOREM 5. Given any first order algebraic difference equation (4.1), there exists a positive number $B$, depending only on the polynomial $P$, such that every continuous solution $u(t)$ of (4.1) satisfies

$$
\liminf _{t \rightarrow+\infty} \frac{|u(t)|}{e_{2}(B t)}=0
$$

and such that every continuous monotonic solution $u(t)$ of (4.1) satisfies

$$
\lim _{t \rightarrow+\infty} \frac{|u(t)|}{e_{2}(B t)}=0
$$

These results are "best possible" in the sense described below.

This theorem was originally proved by Shah [6], but we shall include a proof in this paper in order to show that the result is a special case of Theorems 1 and 3. Shah also pointed out that (4.2) and (4.3) are "best possible" in the following ways. First, it is not possible to replace "lim inf" in (4.2) by "lim" without some restrictive hypothesis, such as monotonicity, on $u(t)$. To show this, let $\phi(t)$ be any real, continuous, increasing function which becomes indefinitely large as $t \rightarrow+\infty$. Let $m$ be any non-negative integer and let $u(t)=t^{m}$ for $t$ in the intervals $[2 n, 2 n+1], n=0,1,2, \cdots$. For $t$ in the intervals $(2 n+1,2 n+2), n=0,1,2, \cdots$, let $u(t)$ be defined in any convenient fashion for which $u(t)$ is continuous and $u$ exceeds $\phi$ at the midpoint. For all $t>0, u(t)$ satisfies an equation of the form (4.1), namely

$$
\left[u(t+1)-(t+1)^{m}\right]\left[u(t)-t^{m}\right]=0 .
$$


Although (4.2) is satisfied, the fact that $u$ exceeds $\phi$ for arbitrarily large $t$ shows that (4.3) cannot be satisfied. Indeed, no relation of the form

$$
\lim _{t \rightarrow+\infty} \frac{|u(t)|}{\phi(t)}=0
$$

is satisfied by all continuous solutions of the particular equation given above, no matter what $\phi(t)$ is.

The equations in (4.2) and (4.3) are also "best possible" in that the denominator $e_{2}(B t)$ cannot be replaced by a function of slower rate of growth. This was proved in $\S 1$ of this paper by considering the equation

$$
u(t+1)-u(t)^{n}=0 .
$$

We shall now show that the relations in (4.2) and (4.3) follow from Theorems 1 and 3. Let $A$ and $C$ be the numbers whose existence was asserted in those theorems, and let $B$ be any number for which $e^{B}>A$. Let

$$
f(t)=e_{2}(B t), \quad g(t)=e^{t}, \quad t>0 .
$$

We need only demonstrate that the hypotheses $\left(\mathrm{H}_{1}\right)$ to $\left(\mathrm{H}_{5}\right)$ are satisfied by $f$ and $g$. It is evident that $\left(\mathrm{H}_{1}\right)$ and $\left(\mathrm{H}_{4}\right)$ are satisfied. In order to establish $\left(\mathrm{H}_{2}\right)$ or $\left(\mathrm{H}_{3}\right)$, we let $b$ be any number greater than zero, $w$ any number greater than one, and $\left\{\tau_{n}\right\}$ any sequence having $\tau_{n}$ in $I_{n}(b)$. Since $I_{n}(b)$ is the interval $[b+n, b+n+1)$, we readily find that

$$
\frac{w^{A^{n}}}{f\left(\tau_{n}\right)} \leqq \frac{w^{A^{n}}}{e_{2}(B b+B n)}=\frac{e\left(A^{n} \log w\right)}{e\left(e^{B b} e^{B n}\right)} .
$$

But since $A<e^{B}$, we have

$$
\lim _{n \rightarrow \infty} \frac{A^{n} \log w}{e^{B b} e^{B n}}=0 .
$$

The relation in (3.4) follows from Lemma 3. In order to establish $\left(\mathrm{H}_{5}\right)$, we let any positive number $\beta$ be given, and put

$$
\alpha=e^{B}, \quad \phi(t)=t, \quad \psi(t)=e^{B t} .
$$

Then

$$
\frac{g(t)^{A^{m+1}}}{f\left(t_{m}\right)}=\frac{e\left(t A^{m+1}\right)}{e\left(e^{B t} e^{B m}\right)}=\frac{e\left\{A^{m+1} \phi(t)\right\}}{e\left\{\alpha^{m} \psi(t)\right\}} .
$$

It readily follows from Lemma 4 that the inequality in (3.14) is satisfied. Thus Theorems 1 and 3 may be applied, with the functions $f$ and $g$ chosen above, and the proof of Theorem 5 is complete.

We can in a similar way obtain a "best possible" result on the rate of increase of solutions of algebraic equations of the form 


$$
P(t, u(t), u(p+q t))=0,
$$

where $p$ and $q$ are real numbers, $q>0, q \neq 1$. In case $p=0$, equations of this type have been called " $q$-difference equations" in the literature. We shall prove the following theorem.

THEOREM 6. Given any first order algebraic q-difference equation (4.10), with $q>0, q \neq 1$, there exists a positive number $B$, depending only on $P, p$, and $q$, such that every continuous solution $u(t)$ of (4.10) satisfies

$$
\liminf _{t \rightarrow+\infty} \frac{|u(t)|}{e\left(t^{B}\right)}=0,
$$

and such that every continuous monotonic solution $u(t)$ of (4.10) satisfies

$$
\lim _{t \rightarrow+\infty} \frac{|u(t)|}{e\left(t^{B}\right)}=0 .
$$

These results are "best possible" in the sense described below.

Proof. The proof of this theorem is similar to that of Theorem 5, and we shall abbreviate the presentation. We first observe that we may without loss of generality assume that $q>1$. For if $0<q<1$, the substitution $\tau=p+q t$ reduces the equation in (4.10) to the form

$$
Q\left(\tau, u\left(q^{\prime} \tau+p^{\prime}\right), u(\tau)\right)=0,
$$

where $q^{\prime}=1 / q>1, p^{\prime}=-p / q$, and $Q$ is also a polynomial with real coefficients. We remark that if $q=1$, the equation in (4.10) is a simple difference equation of the type considered in the preceding section.

As before, the relation in (4.11) is best possible in that it is not possible to replace "lim inf" by "lim" without some restrictive hypothesis, such as monotonicity, on $u(t)$. To show this, we proceed much as before, taking instead of the equation in (4.4) the equation

$$
\left[u(p+q t)-(p+q t)^{m}\right]\left[u(t)-t^{m}\right]=0,
$$

which is of the form in (4.10). Choose any $b$ and let $I_{n}(b)$ have its usual meaning. Let $u(t)=t^{m}$ for $t$ in the intervals $I_{1}, I_{3}, I_{5}, \ldots$. For $t$ in the intervals $I_{0}, I_{2}, I_{4}, \cdots$, let $u(t)$ be defined in any fashion for which $u(t)$ is everywhere continuous. Since this function $u$ satisfies the equation in (4.13) for $t>b$, and is arbitrary in the intervals $I_{0}, I_{2}, I_{4}, \cdots$, no relation of the form in (4.5) can be satisfied by all continuous solutions $u$, no matter what $\phi$ is.

The relations in (4.11) and (4.12) are also "best possible" in that the denominator $e\left(t^{B}\right)$ cannot be replaced by a function of slower rate of growth. For, given any positive integer $n$, and defining

$$
B=\frac{\log n}{\log q},
$$


we see that $e\left(t^{B}\right)$ is a solution of

$$
u(q t)-u(t)^{n}=0,
$$

which is of the form in (4.10).

The equation in (4.10) comes within the scope of the general theory in $\$ 3$, inasmuch as $\delta(t)=p+(q-1) t$ is positive and continuous, and $t+\delta(t)$ is increasing. The relations in (4.11) and (4.12) are again deduced from Theorems 1 and 3. Let $A$ and $C$ be the numbers whose existence was asserted in those theorems, and let $B$ be any positive number for which $q^{B}>A$. Let $D$ be any positive number less than $B$, and take the functions $f$ and $g$ to be

$$
f(t)=e\left(t^{B}\right), \quad g(t)=e\left(t^{D}\right), \quad t>0 .
$$

These functions can be shown to satisfy the hypotheses of Theorems 1 and 3 by a procedure similar to that used above for the functions defined in (4.6). To obtain the analogue of the inequality in (4.7), we observe that if $q^{*}$ is any number satisfying $A<q^{* B}<q^{B}$, then there is a positive number $K$ independent of $n$ for which

$$
b_{n} \geqq K q^{*_{n}} b_{0}, \quad n \geqq 0 .
$$

Hence

$$
f\left(\tau_{n}\right) \geqq e\left(b_{n}^{B}\right) \geqq e\left\{\left(K b_{0}\right)^{B}\left(q^{*}\right)^{n B}\right\} .
$$

Similarly, in obtaining the analogue of (4.9), we use the inequality

$$
t_{m} \geqq q^{* m}, \quad t \geqq T_{1},
$$

and let

$$
\alpha=q^{* B}, \quad \phi(t)=t^{D}, \quad \psi(t)=t^{B} .
$$

In this way, we find that we can apply Theorems 1 and 3 to complete the proof of Theorem 6 .

Finally, we shall apply our general method to the discussion of another class of algebraic functional equations, namely those of the form

$$
P\left(t, u(t), u\left(t^{q}\right)\right)=0
$$

where $q$ is a fixed real number greater than one. Such equations will in this paper be called $q$ th power functional equations. The following theorem provides a "best possible" result on the rate of increase of solutions of such equations.

THEOREM 7. Given any first order algebraic power equation (4.15) with $q>1$, there exists a positive number $B$, depending only on $P$ and $q$, such that every continuous solution $u(t)$ of (4.15) satisfies 


$$
\liminf _{t \rightarrow+\infty} \frac{|u(t)|}{e\left((\log t)^{B}\right)}=0
$$

and such that every continuous monotonic solution satisfies

$$
\lim _{t \rightarrow+\infty} \frac{|u(t)|}{e\left((\log t)^{B}\right)}=0 .
$$

These results are "best possible" in the sense described below.

Proof. The equation in (4.15) comes within the scope of our general theory, since the function $\delta(t)=t^{q}-t$ is positive and continuous, and $t+\delta(t)$ is increasing for $q>1$. It is, perhaps, worth noting that if $0<q<1,(4.15)$ cannot be reduced to the form (1.1) discussed in this paper, except in special cases such as $q=1 / 2$. However, the substitution $\tau=t^{q}$ transforms (4.15) into

$$
P\left(\tau^{r}, u\left(\tau^{r}\right), u(\tau)\right)=0,
$$

where $r=1 / q$. This suggests the consideration of the class of equations of the form

$$
P(t+\delta(t), u(t+\delta(t)), u(t))=0,
$$

wherein $\delta(t)$ is positive and $t+\delta(t)$ is monotonic increasing. A theory of this class of equations parallel to the theory of (1.1) can be constructed, but will be omitted in this article.

Once again it is not possible to replace "lim inf" by "lim" in (4.16) without some restrictive hypothesis. To show this, we choose any $b$, let $u(t)=1$ for $t$ in the intervals $I_{n}(b)$ with odd subscript, and let $u(t)$ be arbitrary in the intervals with even subscript. This function is a solution of an equation of the form in (4.15), namely

$$
\left[u\left(t^{q}\right)-1\right][u(t)-1]=0 .
$$

Moreover, the denominator in (4.16) and (4.17) cannot be replaced by a function of slower rate of growth. For, given any positive integer $n$, if we define $B=(\log n) /(\log q)$, we see that $e\left\{(\log t)^{B}\right\}$ is a solution of the equation

$$
u\left(t^{a}\right)-u(t)^{n}=0 .
$$

The proof of the relations in (4.16) and (4.17) is accomplished as before. We choose any $B$ for which $B>1$ and $q^{B}>A$, choose any $D$ for which $1<D$ $<B$, and let

$$
f(t)=e\left\{(\log t)^{B}\right\}, \quad g(t)=e\left\{(\log t)^{D}\right\}, \quad t>0 .
$$

We shall omit the details, as they are little different from what we have done before.

5. Concluding remarks. In conclusion we may point out that no discussion has been given for equations of higher order such as 


$$
P\{t, u(t), u(t+\delta(t)), u(t+\delta(t)+\delta(t+\delta(t)))\}=0,
$$

since results are necessarily fragmentary. Shah [6] has proved some results for the case $\delta(t)=1$, in which (5.1) becomes

$$
P(t, u(t), u(t+1), u(t+2))=0 .
$$

Nor have we obtained any conditions to ensure the existence of continuous solutions for all $t \geqq a$. Such "nonlocal" existence theorems can be given in certain cases, but an exposition of this point will not be given here. Finally, as remarked previously, a theory of the class of equations of the form

$$
P(t+\delta(t), u(t+\delta(t)), u(t))=0
$$

can be constructed which parallels that for (1.1) given in this paper.

\section{REFERENCES}

1. E. Borel, Mémoire sur les séries divergentes, Ann. Sci. Ecole Norm. Sup. vol. 16 (1899) pp. $9-136$.

2. K. L. Cooke, The rate of increase of real continuous solutions of algebraic differentialdifference equations of the first order, Pacific J. Math. vol. 4 (1954) pp. 483-501.

3. G. H. Hardy, Orders of infinity, Cambridge Tract No. 12, Cambridge University Press, 1910.

4. O. E. Lancaster, Some results concerning the behavior at infinity of real continuous solutions of algebraic difference equations, Bull. Amer. Math. Soc. vol. 46 (1940) pp. 169-177.

5. E. Lindelöf, Sur la croissance des intégrales des équations différentielles algébriques du premier ordre, Bull. Soc. Math. France vol. 27 (1899) pp. 205-215.

6. S. M. Shah, On real continuous solutions of algebraic difference equations, Bull. Amer. Math. Soc. vol. 53 (1947) pp. 548-558.

7. S. M. Shah, On real continuous solutions of algebraic difference equations, II, Proc. Nat. Inst. Sci. India vol. 16 (1950) pp. 11-17.

Pomona College,

Claremont, Calif. 